\title{
Tribological Performance of PTFE-based Coating Modified with Microencapsulated [HMIM][NTf2] Ionic Liquid
}

\author{
Pedro Bandeira ${ }^{1,3}$. Judith Monteiro ${ }^{1}$ ' António Monteiro Baptista ${ }^{2}$ ' Fernão D. \\ Magalhães 3 \\ 1 Flupol, Surface Engineering, Lda, Rua da Central, 401, Campo, 4440-043 Valongo, Portugal \\ 2 CETRIB, Tribology, Vibration and Industrial Maintenance Unit, INEGI, Institute of \\ Mechanical Engineering and Industrial Management, Rua Dr. Roberto Frias, 4200-465 Porto, \\ Portugal \\ 3 LEPABE - Faculdade de Engenharia da Universidade do Porto, Rua Dr. Roberto Frias, 4200- \\ 465 Porto, Portugal
}

\begin{abstract}
In this work, a PTFE-based self-lubricating coating containing microencapsulated [HMIM][NTf2] ionic liquid (IL) lubricant is reported. The microcapsules, made of polysulphone, are prepared by solvent evaporation. In order to allow incorporation in the thin PTFE coating layer, which is applied by spraying, the capsules were produced with small sizes (below 10 $\mathbf{\mu m})$. Their physico-chemical characterization is presented in terms of SEM/EDS, TGA, FTIR and particle size distribution analysis. It is shown that both the encapsulant material and the IL lubricant are able to withstand the high-temperature curing conditions necessary for the coating system used ( $380{ }^{\circ} \mathrm{C}$ during $30 \mathrm{~min}$ ). Crossed-cylinders tribological testing of the applied coatings showed that incorporation of IL-containing capsules yields a reduction in coefficient of friction of up to $12 \%$ when compared to the baseline formulation and a reduction of up to $70 \%$ in wear rate under high load and low sliding speed conditions. The tribological behaviour of the modified coating is further assessed in different load and speed (P.V) combinations.
\end{abstract}

Keywords Microencapsulation $\cdot$ Coating $\cdot$ Ionic liquid $\cdot$ Friction coefficient $\cdot$ Wear rate

\section{Introduction}

Bulk polymer parts and polymer-based coatings are commonly used in tribology, in different contexts. Bulk polymers offer increased durability due to the higher amount of material available for wear, but at the expense of progressive contact misalignment. Polymer coatings make much less material available in the contact area and are therefore less durable, but provide the mechanism with the full rigidity of the substrate materials and prevent major contact misalignments due to the low overall thickness of the coatings. Most of the published work on polymer tribology is concerned with bulk polymers.

High-performance polymeric self-lubricated coatings are in high demand due to increasing 
needs for mechanisms that deliver higher efficiency and robustness. These coatings are often used under harsh tribological conditions such as combinations of dry sliding or starved lubrication with operation under vacuum, high temperature or cryogenic temperature. This poses significant challenges to tribologists and chemists regarding coating development. The concept of a solidliquid self-lubricating coating system $[1,2]$, in which a liquid lubricant is released to the surface as the solid matrix wears out, without the need for external intervention or maintenance, seems interesting in this context, since it may allow significant decrease in friction coefficients and added robustness and durability.

Encapsulation is a logical pathway for the solid-liquid coating concept. Guo and co-workers [3, 4] studied the incorporation of microcapsules containing lubricating agent in bulk epoxy. The microcapsules were produced by in situ polymerization of poly(melamine-formaldehyde) and contained a paraffinic petroleum oil as lubricant. The particle sizes were around $100 \mu \mathrm{m}$. Tribological tests showed effective reduction in friction coefficient. Khun et al. [5] incorporated microencapsulated lubricants, such as paraffin wax, in silicone coatings applied by dipping and then cured, for tribological applications. Armada et al. [1] demonstrated the viability of the solid-liquid coating concept by developing a thermal spray process capable of forming a polyamide coating containing capsules filled with polyalphaolefin lubricant. It must be noted that the thermal spray process used by these authors differs from the spray paint application and curing technique used in the present work.

The aforementioned approaches are limited in terms of maximum service temperature due to the thermal stability of the matrix and lubricant materials used. In addition, use in vacuum conditions is restricted due to the volatility of the liquid lubricants.

In order to cope with a broader envelope of operating conditions, higher-performance materials must be selected as solid-liquid coating constituents. Coating systems formed by a polyamideimide base-coat and a PTFE self- lubricating top-layer are well-proven industrial solutions. But these require high-temperature curing and sintering cycles, in order to form a robust coating that can deliver lubrication properties from cryogenic temperatures up to $260{ }^{\circ} \mathrm{C}$. If a liquid lubricant is to be embedded in such system, it cannot degrade during the high-temperature coating curing step that must typically be performed at $380^{\circ} \mathrm{C}$ for these coatings systems. High thermal stability is paramount for all components of these coating formulations.

Ionic liquids (ILs) consist of a combination of organic cations and, usually, inorganic anions that remain in the liquid state at room temperature. ILs with appropriate combination of a cation and anion have been identified as advanced lubricants in recent years, thanks to their high chemical and thermal stabilities, non-volatility and non- flammability [6,7]. This unique set of properties makes ILs ideal for demanding lubrication applications involving vacuum, high temperature, fire hazards and clean environments operation. In addition, their molecular structure is highly tailorable, theoretically allowing for attaining an optimum balance between relevant properties such as lubrication, thermo-oxidative stability and hydrophobicity [8,9]. Mahrova et al. [10], however, stretch the notion that some care must be taken when using ILs as lubricants due to potentially detrimental effects of by-products from tribomechanical degradation.

Lubrication is one of the wide ranges of tribology parameters [11-13]. Ye et al. [14] were the first to study the lubricating properties of IL anion tetrafluoroborate (BF4) with an imidazolium cation. Minami [9] studied various ILs, observing that the imidazolium type cations are more stable when compared with synthetic oils at $200^{\circ} \mathrm{C}$. He also related the chemical structure of the cations with the tribological properties of ILs, showing that higher alkyl chain imidazolium 
cations directly lead to lower friction coefficients and wear levels. The argument used was that viscosity increases with increasing alkyl chain, and hence, the direct contact between the friction surfaces is more effectively prevented, leading to wear reduction. Somers et al. [6], in an important review on IL lubricants, stressed the capability of anions like PF 6 and BF4 to react with the metal surface and form a protective tribofilm. However, while performing better when compared to lubricating base oils, Minami et al. [8] showed that these anions do not present good hydrolytic stability, which may lead to formation of highly corrosive compounds upon water adsorption, such as hydrofluoric acid (HF). These cause corrosion problems in the lubrication system (tribocorrosion). Subsequent studies have shown that the bis(trifluoromethanesulphonyl)imide (NTf2) anion is more thermally stable and more hydrophobic, avoiding tribocorrosion effects and leading to lower friction coefficient and surface wear [9].

ILs are typically expensive materials, so special attention must be paid to their efficient usage to insure commercial viability of the intended coating. This further stresses the relevance of the encapsulation concept for controlled, on-demand release at the contact surface. However, the works by Sanes et al. [15] and Saur'in et al.

[16] must be mentioned, since these have demonstrated the feasibility of direct incorporation of free IL in polymer matrices (polystyrene, polyamide and epoxy) for enhancement of tribological properties. However, the authors focused their works on performance of bulk polymers rather than coatings. The applicability of this approach in coating formulation is limited not only by the detrimental influence of the IL on the adhesion of the coating to a given substrate, but also by the difficulty in dispersing the IL directly in the waterborne top-coat used in our work and ensuring its homogeneous distribution during the coating curing and stratification stage. These factors can lead to lack of coating cohesion and surface defects.

The information available in the literature is very scarce with regard to techniques for microencapsulation of ILs. Gao et al. [17] used a spray dispersion method followed by sol- vent evaporation to form polysulphone microcapsules containing 1-butyl-3-methylimidazolium hexafluorophosphate [BMIM][PF6] IL. Average size of the microcapsules was around $70 \mu \mathbf{m}$. Xiang et al. [18] produced polyacrylonitrile (PAN) microcapsules containing 1-hexyl-3methylimidazolium tetrafluoroborate [HMIM][BF4], 1-butyl-3-methylimidazolium tetrafluoroborate $[\mathrm{BMIM}][\mathrm{BF} 4]$ and $[\mathrm{HMIM}][\mathrm{PF} 6]$ by solvent extraction in a microfluidic system. The formed microcapsules had an uniform particle size close to $300 \mu \mathrm{m}$. Yang et al. [19] and Chen et al. [20] used polysulphone to encapsulate [BMIM][PF6], also in a microfluidic system, using a solvent evaporation process. The particle sizes produced ranged from 200 to $800 \mu \mathrm{m}$.

The present work describes the use of a solvent evaporation method for encapsulating [HMIM][NTf2] IL in polysulphone. This IL was selected due to the combination of viscosity, hydrophobicity and lubrication capability, as previously studied by Minami [9]. Furthermore, it showed good solubility in the organic phase used in the microencapsulation process. Special care was taken in order to produce small microcapsules (average sizes around $5 \mu \mathrm{m}$ ), so that incorporation in a viable thin tribological coating was not compromised. A coating system composed of a PTFE top-coat and a polyamide-imide/PTFE base-coat was used for evaluating tribological performance. To the best knowledge of the authors, this is the first time that an encapsulated IL is incorporated in a coating for tribological purposes. 


\section{Experimental Section}

\subsection{Materials}

Polysulphone $(\mathrm{PSF}, \mathrm{M} . \mathrm{W} .=75,000)$ was supplied by Acros Organics, 1-hexyl-3methylimidazolium bis(trifluoromethylsulphonyl)imide [HMIM][NTf2] (IL) was supplied by Iolitec GmbH, poly(vinyl alcohol) (PVA, Mowiol 47-88) was kindly provided by Kuraray Europe $\mathrm{GmbH}$, and dichloromethane (DCM, C99.8 \%) was sup- plied by Sigma-Aldrich. For coating system, Dupont 958G-313 one-coat was supplied by Dupont de Nemours and used as primer layer, and 3M Dyneon TF5050Z top-coat was kindly provided by 3M Portugal.

\subsection{Microcapsule Preparation}

The microcapsules were prepared using the solvent evaporation technique. The organic phase was composed of dichloromethane with $4 \%$ by weight ( $w \mathrm{t} \%$ ) of dissolved polysulphone or polysulphone and IL mixture. The aqueous phase consisted on deionized water and $2 \mathrm{wt} \%$ of PVA. In a $1000 \mathrm{ml}$ jacketed reactor, $45 \mathrm{~g}$ of organic phase was dispersed in $270 \mathrm{~g}$ of aqueous phase using an IKA Ultra Turrax T18 rotor-stator at 16,000 rpm, for $30 \mathrm{~min}$ at room temperature. Afterwards, water at $50^{\circ} \mathrm{C}$ (from a thermo- statically controlled bath) was circulated in the reactor jacket to promote solvent evaporation. In order to minimize foam formation, mechanical stirring was initially per- formed with a Cowles impeller at low speed (approximately $50 \mathrm{rpm}$ ). As soon as the initial foam disappeared, the agitation speed was gradually increased to $400 \mathrm{rpm}$, as well as the bath temperature to $70^{\circ} \mathrm{C}$. This evaporation step lasted $2 \mathrm{~h}$. Three different samples of microcapsules were prepared: microcapsules without IL (F01), microcapsules with $33 \mathrm{wt} \%$ of IL (F02) and microcapsules with $67 \mathrm{wt} \%$ of IL (F03).

In order to wash and dry the microcapsules, the final dispersion was centrifuged on a Beckman Avanti J25 device at 12,000 rpm for $30 \mathrm{~min}$. The supernatant was then removed, and the microcapsules were re-suspended in deionized water. This process was repeated three times to ensure complete removal of the PVA from the surface. After this step, the microcapsules were dried at $70^{\circ} \mathrm{C}$ overnight in a convection oven.

\subsection{Physico-chemical Characterization}

Morphology of the microcapsules was observed in a scanning electron microscope with X-ray microanalysis FEI Quanta 400FED ESEM/EDASX Genesis X4M at Centro de Materiais da Universidade do Porto (CEMUP). The dry microcapsules were supported on carbon tape and coated with palladium $(\mathrm{Pd})$ and gold $(\mathrm{Au})$ by sputtering to promote a metallic conductive surface for improved SEM observation.

The particle size distributions (PSD) were measured using a Beckman Coulter LS 230 equipment. Samples were taken for analysis from the final dispersions without further treatment. The computation of the distribution curves was performed with the equipment software, based on the Mie model and using the refraction index of PSF (1.633). The distributions were obtained in triplicate, and the average curve distribution was calculated.

Thermal stability of the microcapsules was analysed on a Netzsch STA449 F3 Jupiter. The 
analysis ranged from 40 to $800{ }^{\circ} \mathrm{C}$ at $10{ }^{\circ} \mathrm{C}$ min $^{-1}$ under inert atmosphere $\left(\mathrm{N}_{2}\right.$, flow rate $30 \mathrm{~mL}$

$\min ^{-1}$ ). The mass of samples was around $5 \mathrm{mg}$, placed in alumina crucibles. For the pure IL analysis, a platinum crucible was used, since the sample was in liquid form.

Infrared (FTIR) spectra were recorded with an ABB Bomem spectrometer, equipped with a Pike MIRacle single reflection ATR accessory. Each spectrum was an average of 32 scans taken with $4 \mathrm{~cm}^{-1}$ resolution in the $4000-500 \mathrm{~cm}^{-1}$ range.

\subsection{Coating Preparation}

A PTFE-based coating system was used for the tribological tests. The system was composed of two coats of Dupont 958G-313 one-coat used as primer (solvent based, com- posed mainly of polyamide-imide binder and PTFE) and one-coat of 3M Dyneon TF5050Z top-coat (waterborne PTFE dispersion, $50 \mathrm{wt} \%$ solids content). The addition of microcapsules was performed by stirring into the waterborne dispersion. The coating systems were applied on the outer face of DIN ISO 355/DIN 720, 30211-A tapered roller bearing tracks (SAE 52100 steel). The track surface was pyrolyzed under air at $400{ }^{\circ} \mathrm{C}$, followed by grit blasting with aluminium oxide flakes to achieve an average surface roughness of $\mathrm{Ra}[3,5] 1 \mathrm{~m}$. The primer was applied by conventional air spray gun and allowed to dry for $10 \mathrm{~min}$ at $80{ }^{\circ} \mathrm{C}$ in a convection oven. The top-coat was then applied over the dried primer using a conventional air spray gun and allowed to dry for a further $10 \mathrm{~min}$ at $80^{\circ} \mathrm{C}$ in a convection oven. The final step was the coating cure, performed in a convection oven at $380{ }^{\circ} \mathrm{C}$ for $30 \mathrm{~min}$. The final thickness of the coatings was approximately $30 \mathrm{\mu m}$ measured with an Elcometer 355 coating thickness gauge. It must be noted that the convex shape of the test tracks does influence the dry film thickness (DFT) value measured by such equipment, effectively underestimating it, as can be confirmed with the SEM images of the coating cross sections. This is a well-known fact in the coatings industry and is accepted as a standard as long as the measurement equipment is properly calibrated. For this study, Coating A was produced as a baseline reference containing no microcapsules. Coatings $\mathrm{B}, \mathrm{C}$ and $\mathrm{D}$ were prepared with the same base materials as Coating A, but the top-coat was modified with $5 \mathrm{wt} \%$ of microcapsules F01, F02 and F03, respectively.

\subsection{Tribological Testing}

Tribological testing was performed on a crossed-cylinders type tribometer built at CETRIB. The tribometer is capable of operating in the $[12 ; 1500] \mathrm{N}$ load range due to its construction philosophy, and the operating speed range is $[40 ; 500] \mathrm{rpm}$ with the actual contact sliding speed being dependent on the diameter of the test track. Rotating speed is controlled in closed loop, and an encoder is used to record the number of rotation cycles for each test. We then use the diameter of the test track to extrapolate the sliding length for each test.

Friction force is measured directly with a load cell, and the measurements are acquired through a National Instruments card directly into LabView software. The test specimens consisted of outer tracks of standard 30211-A tapered roller bearings (SAE 52100 steel) with the coating applied on the external faces of the tracks, and the counterface specimens consisted of standard bearing rollers (SAE 52100 steel) with $5 \mathrm{~mm}$ diameter and $19.6 \mathrm{~mm}$ length. The contact area resulting 
from this test setup is a theoretical single point that provides convenient self- aligning capability combined with high Hertzian contact pressure. Figure 1 shows the crossed-cylinder configuration scheme

The tribometer performs real-time data acquisition of the friction force during testing, which is converted into a reading of coefficient of friction $(\mathrm{COF})$ via Eq. 1, where $(l)$ is the COF, ( $F$ friction) is the friction force, and $(L)$ is the applied load.

$$
\mu=\frac{F_{\text {friction }}}{L}
$$

Tribological testing parameters were varied at four different load and speed (P.V) combinations according to Table 1 to allow correct assessment of coating performance. Each trial curve plotted in this work is an average of three repetitions.

Wear rate is measured according to Eq. 2, where $(W)$ is the wear rate $\left(\mathrm{mm}^{3} \mathrm{~N}^{-1} \mathrm{~m}^{-1}\right),(V)$ is the removed material volume $\left(\mathrm{mm}^{3}\right),(d)$ is the sliding length $(\mathrm{m})$, and $(L)$ is the applied load $(N)$. To measure the removed material volume, a representative section of the tested track was analysed by a mechanical stylus profilometer (Hömmel Tester T4000 bench top) along a $500 \mu \mathrm{m}$ length of each test track. The device is equipped with a precision $\mathrm{x}-\mathrm{y}$ table allowing the scan of the wear tracks by measuring a series of roughness profiles taken $5 \mu \mathrm{m}$ apart. For the presented measurements, 101 roughness profiles were taken for each measured sample and then transformed in a 3D surface map by polynomial interpolation of these profiles using the Hömmel Map software supplied with the machine. Using these measurements and establishing that the wear profile of the complete track perimeter is identical, it is possible to extrapolate the removed material volume to the total perimeter of the test track. The sliding length (d) was kept constant for all tests, and applied load $(L)$ and sliding speed were varied in four distinct sets al- lowing for a good characterization of the coating system behaviour. A second tribological test type was performed varying the sliding distance while keeping a chosen combination of load and speed constant, to allow the assessment of wear rate evolution along the test duration and thus support the unusual behaviour of the running-in phase on the main tests. The assumption that the wear profile is constant along the perimeter was verified by performing topography measurements on previously tested tracks, taken at four sites separated by $90^{\circ}$ on each track. Since no statistically significant differences were found, the assumption of test track wear profile homogeneity was deemed to hold true. Each wear rate measurement presented in this work is the average of three separate measurements, performed, respectively, on each of the three repeated tracks used for tribological test repeatability verification.

\section{Results and Discussion}

\subsection{Microcapsules Characterization}

Figure 2 shows SEM images of the microcapsules produced with different IL contents. The capsules surface is smooth and homogeneous in all cases. There is a broad range of diameters, 
but the maximum value is always around $5 \mu \mathrm{m}$. The difference in IL:PSF ratio does not produce significant changes on size and external appearance of the microcapsules.

Figure 3 shows the PSD measured for the final dispersions. The distributions are bimodal, with the larger volume fraction lying in the $0-9 \mu \mathrm{m}$ range. The secondary peaks correspond to sizes in the 10-25 $\mathrm{\mu m}$ range, having no correspondence with actual particles observed in SEM images, and are therefore believed to be agglomerates of smaller capsules. It is apparent from Fig. 3 that the fraction of these agglomerates tends to increase when IL is used, probably due to residual presence in the outer surface promoting adhesion between particles. Such agglomerates were never visible in the final coatings, either by visually or by SEM imaging. This may be due to the surfactant system present in the waterborne PTFE dispersion contributing for deagglomeration and stabilization of the microcapsules upon mixing of the two dispersions. In addition, shear stresses during spraying of the liquid coating may also induce deagglomeration. Energy-dispersive X-ray spectroscopy (EDS) was performed on previously washed and dried microcapsules. The electron beam used allows for a 2-3 $\mu \mathrm{m}$ penetration in the analysed surface. Figure 4 presents the EDS spectra of the capsules. Carbon (C), oxygen (O) and sulphur (S) are present both in the capsule shell and in the IL and are therefore observed in both Fig. 4a and b. Fluorine (F), on the other hand, exists only in the IL, and its characteristic peak can be seen in Fig. $4 \mathrm{~b}$, confirming its presence in the microcapsules.

Figure 5 shows FTIR spectra of the microcapsules and IL. Microcapsules spectra (F01, F02 and F03) show characteristic bands of PSF at: 1583,1503 and $1486 \mathrm{~cm}^{-1}(\mathrm{C}=\mathrm{C}$ aromatic rings stretching) [21], 1322 and $\sim 1293 \mathrm{~cm}^{-1} \quad(\mathrm{~S}=\mathrm{O}$ sulphone asymmetric stretching) [22], $1234 \mathrm{~cm}^{-1}$ (C-O-C stretching) [23], and $\sim 832, \sim 853$ and $\sim 873 \mathrm{~cm}^{-1}$ ( $q$ disubstituted benzene ring) [21]. The spectra of microcapsules containing IL (F02 and F03) and of free IL show characteristic bands at: $\sim 1349 \mathrm{~cm}^{-1}$ ( $\mathrm{S}=\mathrm{O}$ asymmetric stretching) [22], $\sim 1180 \mathrm{~cm}^{-1}$ (CF3 asymmetric stretching) [22], $\sim 1054 \mathrm{~cm}^{-1}$ (S-N-S asymmetric stretching) [22], $\sim 615 \mathrm{~cm}^{-1}$ (NTf2 trans-isomer) [24], and $\sim 655 \mathrm{~cm}^{-1}$ and $\sim 600 \mathrm{~cm}^{-1}$ (NTf2 cis-isomer) [24]. In spectra F02 and F03, the intensity of all bands associated with IL (zones b, c and e in Fig. 5) relative to those from PSF (zones a and d in Fig. 5) is seen to increase with IL content. This set of EDS and FTIR data confirms encapsulation of IL in PSF particles. For support, the chemical structures of IL and PSF are presented in Fig. 6a and b, respectively.

The thermal degradation of microcapsules with and without IL was analysed by thermogravimetric analysis. The results are shown in Fig. 7. The extrapolated onset temperature for microcapsules of PSF alone (F01) is around $514{ }^{\circ} \mathrm{C}$, in agreement with literature values for PSF decomposition $[17,25]$. About $26 \%$ of the sample remains at $800{ }^{\circ} \mathrm{C}$, indicating formation of thermally stable carbonaceous material, probably associated with the presence of aromatic structures in the polymer backbone. Free (non- encapsulated) IL shows degradation onset temperature at $408^{\circ} \mathrm{C}$, in agreement with a previously reported value [26]. Microcapsules F02, with IL:PSF ratio 1:2, degrade in two steps. The first is expected to correspond to thermal degradation of the IL. The corresponding onset temperature $\left(420{ }^{\circ} \mathrm{C}\right)$ is higher than the value obtained for free IL, since the encapsulating PSF shell acts as a protective thermal barrier and retards escape of volatile decomposition products. The second step is not well defined and shows an onset temperature of about $460^{\circ} \mathrm{C}$, lower than for PSF alone. Microcapsules F03, with IL:PSF 
ratio $1: 1$, show an onset temperature of $418^{\circ} \mathrm{C}$, similar to $\mathrm{F} 02$ and corresponding to degradation of encapsulated IL. However, the second degradation step, corresponding to PSF degradation, is apparently superimposed with the first. This progressive shift of PSF degradation towards lower temperatures with increasing IL content is probably a consequence of facilitated decomposition of PSF in the presence of sulphuric and hydrofluoric acids that result from the thermal degradation of this IL [27] as a direct consequence of the higher IL:PSF ratio.

The percentage of stable residues at $800{ }^{\circ} \mathrm{C}$ is proportional to the amount of PSF in the formulations. The experimental values for F02 and F03 are 19.1 and $11.1 \%$ by weight, respectively. This agrees reasonably well with the expected values of 17.4 and $13.1 \%$, computed based on the residue obtained for F01 $(26.1 \%)$ and the PSF fraction in the other two formulations.

The results from the TGA analysis reveal that some care must be taken in the usage of PSFencapsulated [HMIM][NTf2] IL near the decomposition onset temperature $\left(420{ }^{\circ} \mathrm{C}\right)$, due to predictable release of sulphuric and hydrofluoric acids. These can pose, among others, maintenance problems in the tribological mechanisms due to chemical corrosion. This limitation, however, is well understood by users of PTFE-based coatings, since this material is also capable of releasing hydrofluoric acid upon degradation at temperatures over $400^{\circ} \mathrm{C}$.

\subsection{Coating Characterization}

Several coatings were prepared for testing in terms of tribological performance. Coating A contained no microcapsules, while Coatings B, C and D contained microcapsule formulations F01, F02 and F03, respectively. Figure 8 depicts a cross section of an unworn test track for each of the produced samples, shown in secondary electron mode (SE) and backscattered electron mode (BS), after fracture under liquid nitrogen.

The distinction between the steel track and the coating materials is clearly outlined in BS mode, as well as the coating stratification due to different compositions of the primer and top-coats.

Figure 9 depicts a cross section of a region within the worn test track for each of the produced samples after a $2000 \mathrm{~m}$ sliding length (see tribological testing section). These images are shown in perspective for a clearer view of the worn track surface morphology. As can be seen, all coatings still present a very significant thickness in the worn area, even after $2000 \mathrm{~m}$ of sliding.

Figure 10 shows a magnification of a coating cross section, showing the presence of the microcapsules in the PTFE top- coat and the EDS spectra for the different areas, evidencing the expected different elemental composition between the polysulphone microcapsules containing IL $(\mathrm{C}, \mathrm{O}, \mathrm{S}$ and $\mathrm{F})$ and the top-coat PTFE material $(\mathrm{C}, \mathrm{O}$ and $\mathrm{F})$. These observations confirm that the microcapsules maintained their integrity during the high-temperature coating curing step.

\subsection{Tribological Testing}

The measured COF as a function of sliding length for the different load and speed (P.V) combinations is shown in Fig. 11, for all the formulated coatings, and shows the P.V combination used for the respective tests.

It is worth mentioning that PSF is mechanically much harder than the PTFE solid lubricant and additionally presents a much higher COF against steel. This remark makes the interpretation of friction and wear phenomena better understandable. 
For P.V1 (Fig. 11a), it is clear that the addition of microcapsules has a slightly detrimental effect on the COF for all cases including the microcapsules with IL, mainly due to the presence of PSF as the microcapsule structure material. The presence of PSF microcapsules in the coatings constitutes a foreign body to the original tribo-pair. The to the presence of PSF as the microcapsule structure material. The presence of PSF microcapsules in the coatings constitutes a foreign body to the original tribo-pair. The IL-containing microcapsules are still able to slightly de- crease this detrimental impact, and it can be seen that Coating D performs the best in the microcapsule-containing coatings group due to the higher IL contents and probably due to the residual presence of IL outside the microcapsules.

For P.V2 (Fig. 11b), the effect of microcapsules addition is similar to what was observed for P.V1, but clearly the higher sliding speed was determinant for the IL not being able to play a COF reducing role.

It is important to state that at a load level of $12 \mathrm{~N}$, the rupture of the microcapsules is a very lengthy process, which has an effect on the IL release rate. At $400 \mathrm{~mm} . \mathrm{s}^{-1}$ sliding speed, the release rate of the IL can be sufficient to promote the availability of enough material to form a semi- boundary-layer effect that can explain the better behaviour of the IL-containing microcapsules, but at a sliding speed of $800 \mathrm{~mm} \mathrm{~s}^{-1}$, the IL release rate is clearly insufficient to allow the availability of enough material to form this layer, thus rendering the presence of IL irrelevant with respect to the COF measurements.

At P.V3 (Fig. 11c), the foreign body effect of the PSF microcapsules is well perceived with the curve of Coating B, but Coatings $\mathrm{C}$ and $\mathrm{D}$ show that the IL is able to overcome this effect and bring appreciable benefits regarding the tribo-pair's COF. At $75 \mathrm{~N}$ load level, there is enough stress in the contact to promote a faster rupture of the IL-containing microcapsules. This effect makes it possible for the IL to be present in the contact area in sufficient quantity to form an effective boundary lubrication layer that effectively reduces the COF despite the influence of the PSF residues. It is noticeable that the difference in IL contents in the microcapsules of Coatings $\mathrm{C}$ and $\mathrm{D}$ did not produce a discernible distinction in performance regarding measured COF, therefore indicating that at P.V3 and for the tested sliding duration, the quantity of IL available from the F02 microcapsules is sufficient to produce the desired effect, and the superior IL quantity used for the F03 microcapsules does not bring additional benefit.

For P.V4 (Fig. 11d), the effect of PSF microcapsules without IL is similar to the one observed for P.V3, and at a fist glance, the IL-containing microcapsules just seem to be able to overcome the negative impact of PSF foreign body effect without bringing added benefit when compared to Coating A. A closer look at the data, however, reveals that after approximately $1250 \mathrm{~m}$ of sliding distance, the presence of IL on Coatings $\mathrm{C}$ and $\mathrm{D}$ produces a reduction in COF. A possible explanation for this phenomenon might be connected to the IL release rate to the contact area, which might not be fast enough to immediately make sufficient IL available to form a boundary lubrication layer. After the stated $1250 \mathrm{~m}$ of sliding distance, the quantity of IL present in the contact area builds up to the point where the boundary layer is actually formed, and the beneficial effects of the IL start to produce visible results.

Topography profiles of the tested wear tracks are shown in Fig. 12 for P.V3 as an illustrative example of the methodology used for all cases. It is possible to see differences between the coatings in terms of surface rough- ness and depth of the wear track. These topographic profiles also allow measurements of removed material volume $(V)$ during the tests and provide the 
necessary in- formation to compute wear rates $W$ according to Eq. 2. The first conclusion to be taken from the topographic analysis is that the initial surface roughness of all coatings is in the order of $3 \mathrm{~lm} \mathrm{Ra}$, which immediately lends itself to initial wear, during the running-in phase, significantly higher than that observed after the smoothening of the roughness peaks. This has been previously observed by Solzak and Polycarpou [28] in published works involving commercial PTFE-based coatings, which helps to under-stand that the running-in phase for this type of coatings is very rapid and does not present a typical behaviour. The initial roughness on Coating A (region outside the wear track) is apparently lower in the topographies shown in Fig. 12 , but this is only due to the scale compression effect introduced by the height of accumulated material on the edges of the worn track. This material accumulation is a direct consequence of material flow during the tribological test. Since the magnitude of this behaviour for the remaining tested coatings is either much smaller or inexistent (case of Coating B), a conclusion can readily be drawn towards the PSF microcapsules playing a role on increasing the load-bearing capacity of the base-coat, which in turn results in higher wear resistance.

Wear rate analyses are based on the information shown in Fig. 13 that depicts the measured wear rates for all coatings under the different P.V conditions. For P.V1, Coating B stands out due to a lower wear resistance. This phenomenon might be explained due to the low loading conditions and low speed combination favouring an adhesive behaviour between the counterface pin and the PSF microcapsules, tearing the microcapsules from the coating and making them act as an abrasive third body in the contact area, therefore accelerating the wear process. Coatings $\mathrm{C}$ and D show a wear resistance performance that is only slightly better than Coating A, probably due to the fact that the IL disables the proposed adhesive mechanism between the PSF microcapsules and the counterface pin, originating a slight overall benefit due to the hardness of the PSF microcapsules. This hypothesis is in agreement with the presented discussion for the COF analyses.

At P.V2 conditions, the contribution of microcapsules appears to be much more relevant in the increase of wear resistance. Coating $B$ readily presents a significant reduction in wear rate compared with Coating A, still with no presence of IL. This phenomenon might be explained by the disabling of the adhesive mechanism between the counterface pin and the PSF claimed for the P.V1 conditions, due to the higher sliding speed. This disabling might be further studied in the future with intermediate variations of sliding speed. Coatings $\mathrm{C}$ and $\mathrm{D}$ present further improvements in wear resistance, indicating a beneficial effect of the IL in these test conditions. The error bars are shown for these coatings; however, the IL available in the contact area is not sufficient to produce a sustained effect and is in agreement with the observations made for the COF analyses.

Under P.V3 and P.V4 test conditions, the effect of the microcapsules is very noticeable in terms of wear reduction, and this is mainly attributed to the hardness of the PFS microcapsule structure. Compared to Coating A, all other coatings show at least a $50 \%$ reduction in wear rate for P.V3 and at least $70 \%$ reduction in wear rate for P.V4. Theeffect of the IL itself is not shown to have a significant contribution to wear resistance increase under these test conditions.

Figure 14 a-d shows the SEM images of counterface steel rollers used, respectively, in the testing of Coatings $\mathrm{A}, \mathrm{B}, \mathrm{C}$ and $\mathrm{D}$ at P.V3.

The observed counterface wear area is in good agreement with the analysis of friction behaviour for the different coatings. Use of the unmodified coating (A) results in the highest wear area. Addition of PSF microcapsules (Coating B) shows a decrease in worn area, due to in- creased 
load-bearing capability. Coatings $\mathrm{C}$ and $\mathrm{D}$ produce the smallest counterface wear areas due to the action of the IL lubrication. The presence of IL seems to reduce the abrasiveness of the contact, supporting the hypothesis of a boundary lubrication film formation.

The evolution of wear with respect to sliding length was also analysed for Coatings A and D at P.V3. The resulting graphics are shown in Fig. 15a and b. Both coatings present an initially high wear rate that corresponds to the running- in phase. Coating D actually shows an initially higher wear rate up to approximately $125 \mathrm{~m}$ of sliding distance. This is attributed to the rupture of nearsurface microcapsules that disturb the run-in process, with PSF residues possibly acting as a counter-productive third body in the contact zone. From about 250-m sliding distance onwards, the contribution of the IL towards wear reduction starts to dominate. At $2000 \mathrm{~m}$ sliding distance, the differences be- tween the two coatings are substantial. It is important to understand that heat continuously builds up during a trial, and from $1000 \mathrm{~m}$ of sliding onwards, the temperature can have an effect in the acceleration of wear in the coatings. This effect can explain the final increase in wear rate and removed material volume for Coating A. As this is not observed in Coating D, we believe it constitutes further evidence of the IL's beneficial action in the tribological pair.

\section{Conclusions}

- An effective methodology for preparation of PSF microcapsules containing [HMIM][NTf2] IL with mean spherical dimensions around $5 \mu \mathrm{mm}$ is presented.

- The proposed solvent evaporation method is simple and readily scalable for production of large quantities of microcapsules, configuring an attractive route for industrial exploitation without requiring major investments.

- The microcapsules were successfully incorporated in thin PTFE-based coatings ( $5 \mathrm{wt} \%$ loading), thanks to the small microcapsules size and the ability of PSF and IL to withstand the temperature conditions necessary for coating cure.

- The presence of PSF microcapsules containing IL was shown to be beneficial for the measured COF under high loading conditions combined with low or high sliding speeds. On the other hand, the microcapsules were slightly detrimental under low loading conditions combined with low or high sliding speeds.

- Wear resistance of the coatings modified with PSF microcapsules containing IL was shown to be much improved relatively to the baseline coating.

- The hypothesis of an IL boundary lubrication film being formed at a load level of $75 \mathrm{~N}$ and $400 \mathrm{~mm} \mathrm{~s}^{-1}$ sliding speed was supported by the decreasing abrasive effect observed on the counterface pin with the increase in IL concentration.

\section{Acknowledgments}

This work was funded by Flupol Surface Engineering Lda and by FEDER funds through the Operational Programme for Competitiveness Factors - COMPETE, ON.2 - O Novo NorteNorth Portugal Regional Operational Programme and National Funds through FCT - Foundation for Science and Technology under the projects: PEst-C/EQB/UI0511, NORTE-07-0124-FEDER000026-RL1_Energy. The authors would also like to acknowledge Flupol for performing the technical application of all coatings on the tested tracks. 
Conflict of interest Author P. Bandeira has received a Ph.D. Grant from Flupol.

\section{References}

1. Armada, S., Schmid, R., Equey, S., Fagoaga, I., Espallargas, N.: Liquid-solid self-lubricated coatings. J. Therm. Spray Technol. 22, 10-17 (2013)

2. Espallargas, N., Vitoux, L., Armada, S.: The wear and lubrication performance of liquidsolid self-lubricated coatings. Surf. Coat. Technol. 235, 342-353 (2013)

3. Guo, Q.B., Lau, K.T., Zheng, B.F., Rong, M.Z., Zhang, M.Q.: Imparting ultra-low friction and wear rate to epoxy by the in- corporation of microencapsulated lubricant? Macromol. Mater. Eng. 294, 20-24 (2009)

4. Guo, Q.B., Lau, K.T., Rong, M.Z., Zhang, M.Q.: Optimization of tribological and mechanical properties of epoxy through hybrid filling. Wear 269, 13-20 (2010)

5. Khun, N.W., Zhang, H., Yang, J.L., Liu, E.: Tribological performance of silicone composite coatings filled with wax-containing microcapsules. Wear 296, 575-582 (2012)

6. Somers, A., Howlett, P., MacFarlane, D., Forsyth, M.: A review of ionic liquid lubricants. Lubricants 1, 3-21 (2013)

7. Bermudez, M.D., Jimenez, A.E., Sanes, J., Carrion, F.J.: Ionic liquids as advanced lubricant fluids. Molecules 14, 2888-2908 (2009)

8. Minami, I., Kita, M., Kubo, T., Nanao, H., Mori, S.: The tribological properties of ionic liquids composed of tri- fluorotris(pentafluoroethyl) phosphate as a hydrophobic anion. Tribol. Lett. 30, 215-223 (2008)

9. Minami, I.: Ionic liquids in tribology. Molecules 14, 2286-2305 (2009)

10. Mahrova, M., Conte, M., Roman, E., Nevshupa, R.: Critical insight into mechanochemical and thermal degradation of imidazolium- based ionic liquids with alkyl and monomethoxypoly(ethylene glycol) side chains. J. Phys. Chem. C 118, 22544-22552 (2014)

11. Neale, M.: Tribology Handbook. Elsevier, Amsterdam (1996)

12. Holmberg, K., Matthews, A.: Coatings Tribology: Properties, Mechanisms, Techniques and Applications in Surface Engineering. Elsevier, Amsterdam (2009)

13. Xie, Y-B.: Theory of Tribo-Systems. In: Kuo, C-H. (ed.) Tribology - Lubricants and Lubrication, pp. 3-32. InTech (2011)

14. Ye, C., Liu, W., Chen, Y., Yu, L.: Room-temperature ionic liquids: a novel versatile lubricant. Chem. Commun. 0, 2244-2245 (2001)

15. Sanes, J., Carrion, F.J., Bermudez, M.D., Martinez-Nicolas, G.: Ionic liquids as lubricants of polystyrene and polyamide 6-steel contacts. Preparation and properties of new polymer-ionic liquid dispersions. Tribol. Lett. 21, 121-133 (2006)

16. Saurín, N., Sanes, J., Bermúdez, M.D.: Effect of graphene and ionic liquid additives on the tribological performance of epoxy resin. Tribol. Lett. 56, 133-142 (2014)

17. Gao, H.S., Xing, J.M., Xiong, X.C., Li, Y.G., Li, W.L., Liu, Q.F., et al.: Immobilization of ionic liquid BMIM PF6 by spraying suspension dispersion method. Ind. Eng. Chem. Res. 47, 4414-4417 (2008)

18. Xiang, Z.Y., Lu, Y.C., Zou, Y., Gong, X.C., Luo, G.S.: Preparation of microcapsules containing ionic liquids with a new sol- vent extraction system. React. Funct. Polym. 68, 1260- 
1265 (2008)

19. Yang, W.W., Lu, Y.C., Xiang, Z.Y., Luo, G.S.: Monodispersed microcapsules enclosing ionic liquid of 1-butyl-3-methylimida- zolium hexafluorophosphate. React. Funct. Polym. 67, 8186 (2007)

20. Chen, D.X., OuYang, X.K., Wang, Y.G., Yang, L.Y., Yu, D.: Polysulfone microcapsules containing ionic liquid. Adv. Mater. Res. 554 - 556, 273-276(2012)

21. Chalmers, J., Griffiths, P.: Handbook of Vibrational Spectroscopy. Wiley, Hoboken (2001)

22. Rey, I., Johansson, P., Lindgren, J., Lassègues, J.C., Grondin, J., Servant, L.: Spectroscopic and Theoretical Study of (CF3SO2)2N- (TFSI-) and (CF3SO2)2NH (HTFSI). J. Phys. Chem. A 102, 3249-3258 (1998)

23. Dizman, C., Ates, S., Torun, L., Yagci, Y.: Synthesis, characterization and photoinduced curing of polysulfones with (meth)acrylate functionalities. Beilstein J. Org. Chem. 6, 56 (2010)

24. Paulechka, Y.U., Kabo, G.J., Blokhin, aV, Shaplov, aS, Lozinskaya, E.I., Golovanov, D.G., et al.: IR and X-ray study of polymorphism in 1-alkyl-3-methylimidazolium bis(trifluoromethanesulfonyl)imides. J. Phys. Chem. B 113, 9538-9546 (2009)

25. Li, X.-G., Huang, M.-R.: Thermal degradation of bisphenol A polysulfone by high-resolution thermogravimetry. React. Funct. Polym. 42, 59-64 (1999)

26. Tokuda, H., Hayamizu, K., Ishii, K., Susan, M.A.B.H., Watanabe, M.: Physicochemical properties and structures of room tem- perature ionic liquids. 2. Variation of alkyl chain length in imidazolium cation. J. Phys. Chem. B 109, 6103-6110 (2005)

27. Maton, C., De Vos, N., Stevens, C.V.: Ionic liquid thermal stabilities: decomposition mechanisms and analysis tools. Chem. Soc. Rev. 42, 5963-5977 (2013)

28. Solzak, T.A., Polycarpou, A.A.: Tribology of WC/C coatings for use in oil-less piston-type compressors. Surf. Coat. Technol. 201, 4260-4265 (2006)

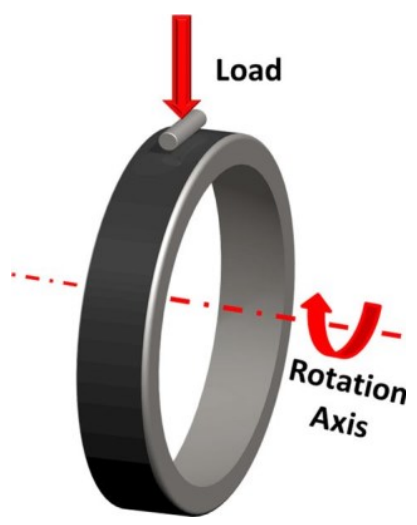

Fig. 1 Crossed-cylinders configuration scheme 

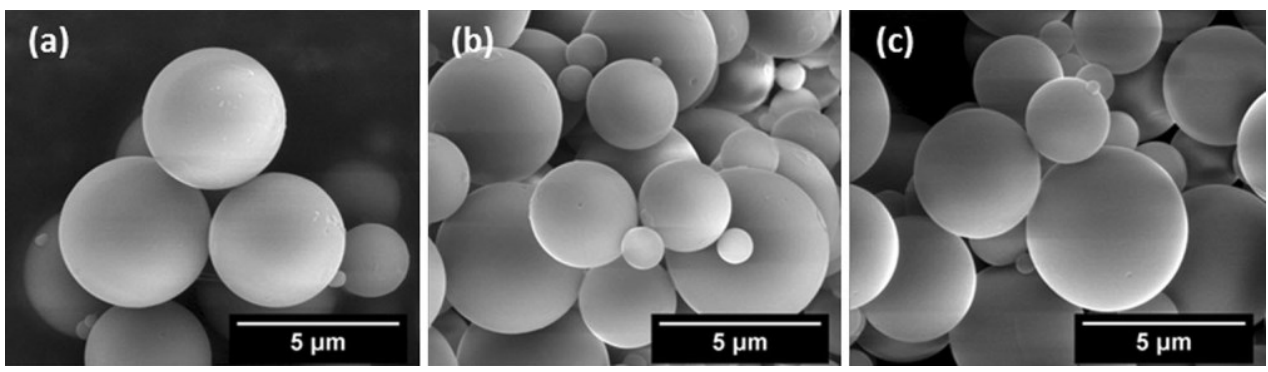

Fig. 2 SEM images of microcapsules. Magnification: 20,000X. The different images correspond to formulations F01 (a), F02 (b) and F03 (c)

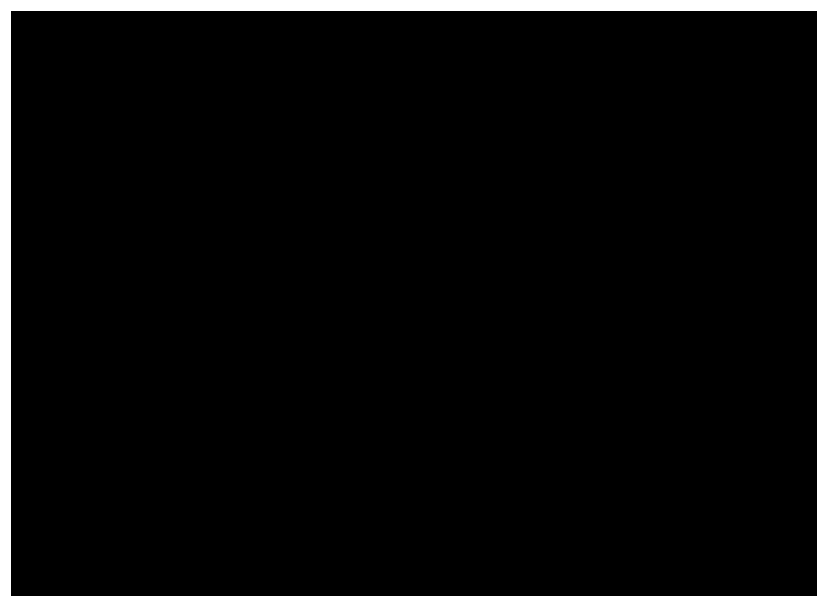

Fig. 3 Differential volume particle size distribution (PSD) of micro- capsule formulations 


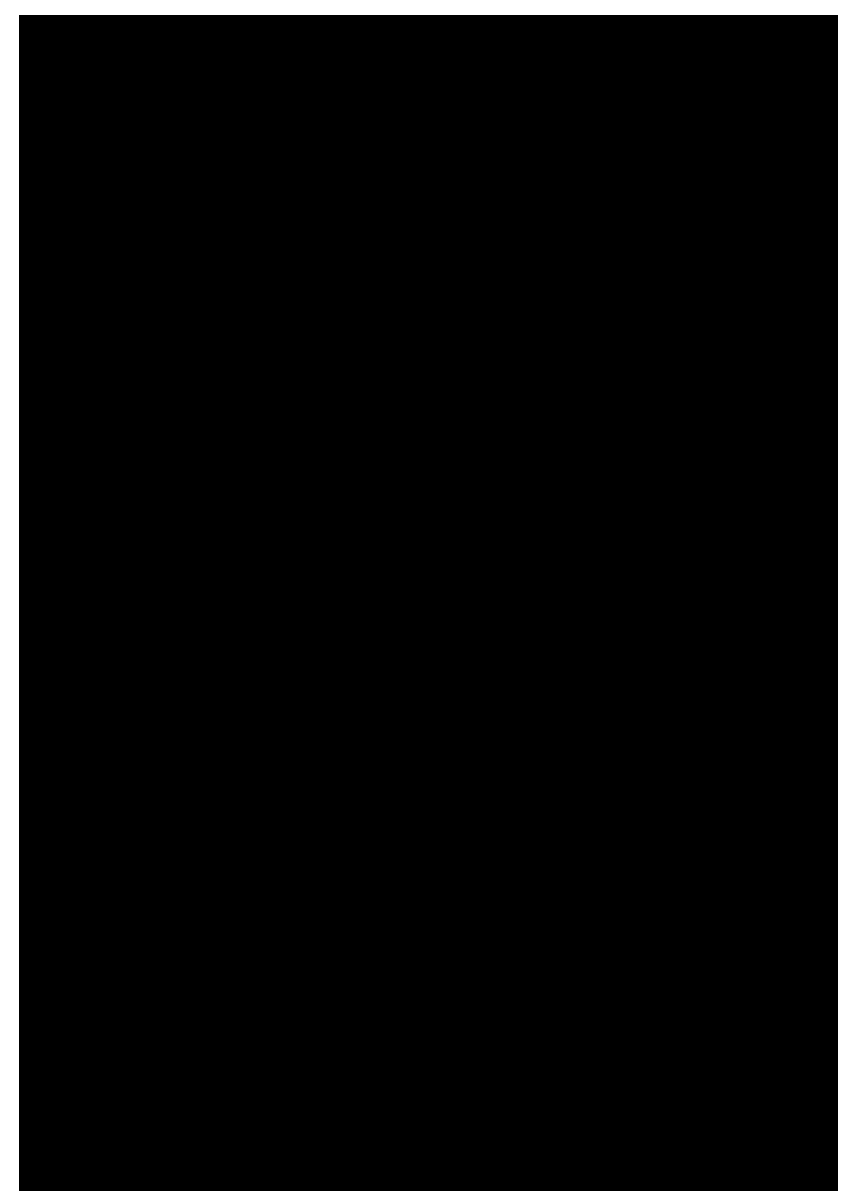

Fig. 4 EDS spectra of microcapsules: F01 (a), F02 and F03 (b)

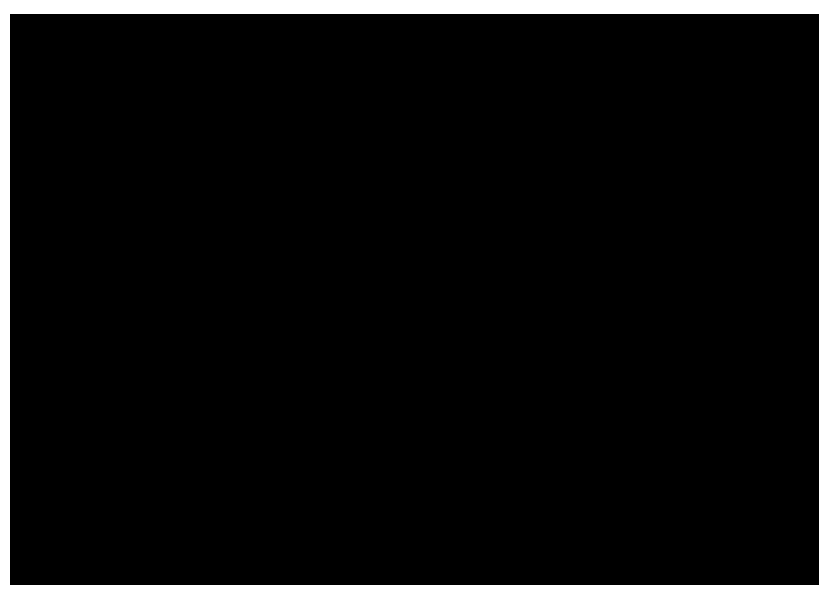

Fig. 5 FTIR-ATR spectra of microcapsule formulations (F01, F02 and F03) and IL. Zones $a$ and $d$ : characteristic bands of PSF; zones $b, c$ and $e$ : characteristic bands of IL 


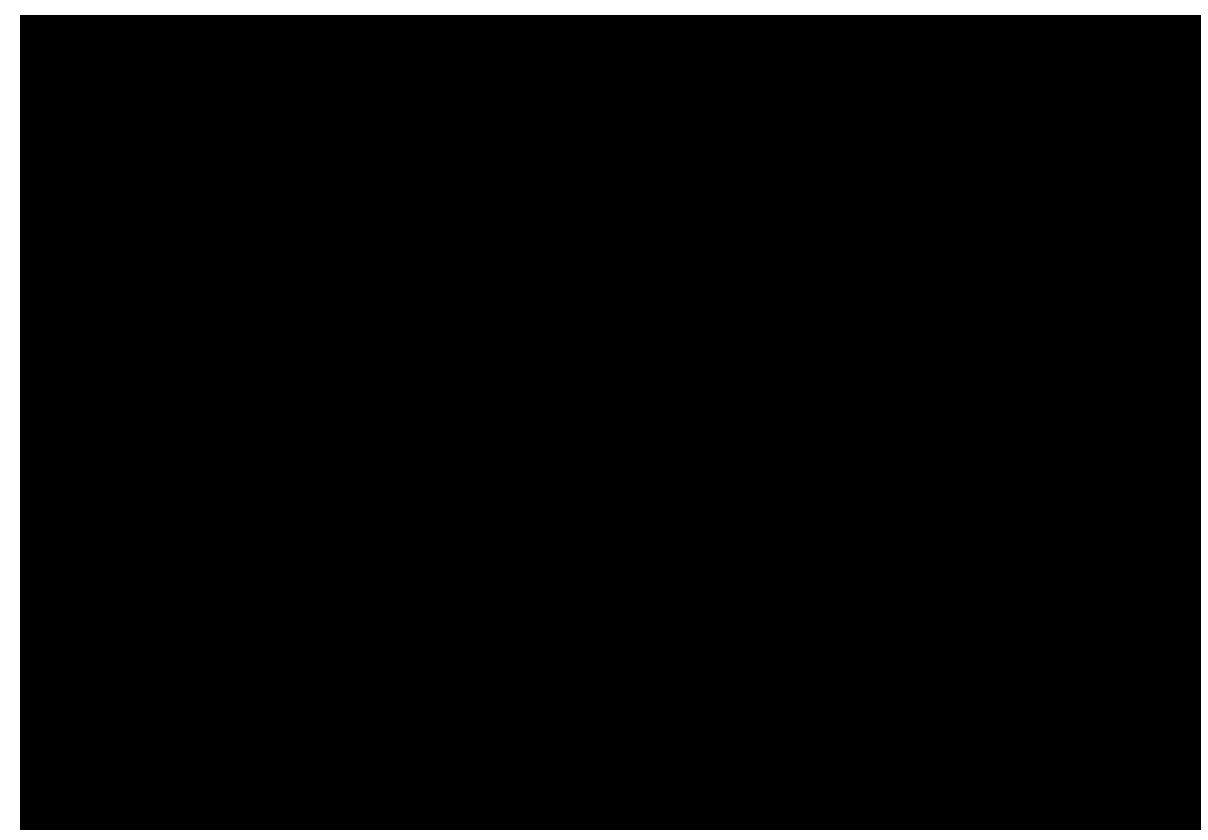

Fig. 6 Molecular structure of ionic liquid [HMIM][NTf2] (IL) (a) and polysulphone (PSF) (b) molecular structure

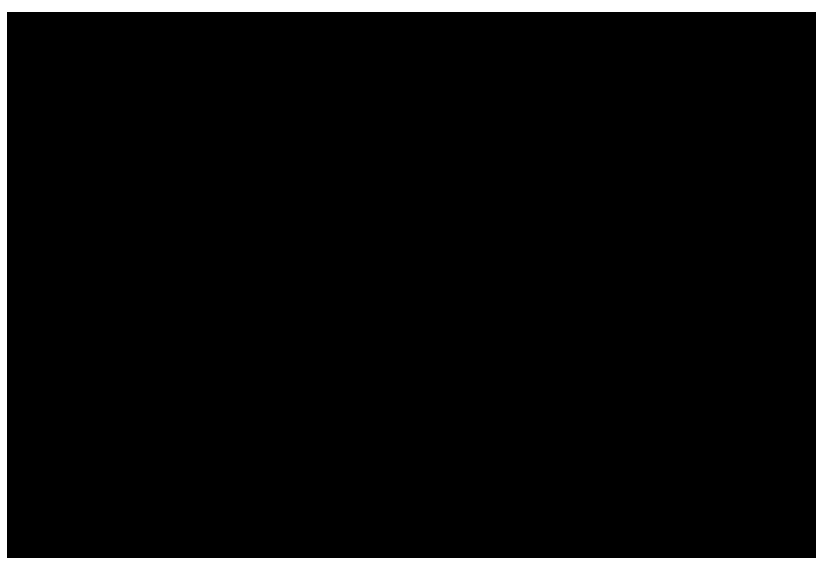

Fig. 7 Thermogravimetric curves obtained for free ionic liquid and microcapsule formulations 


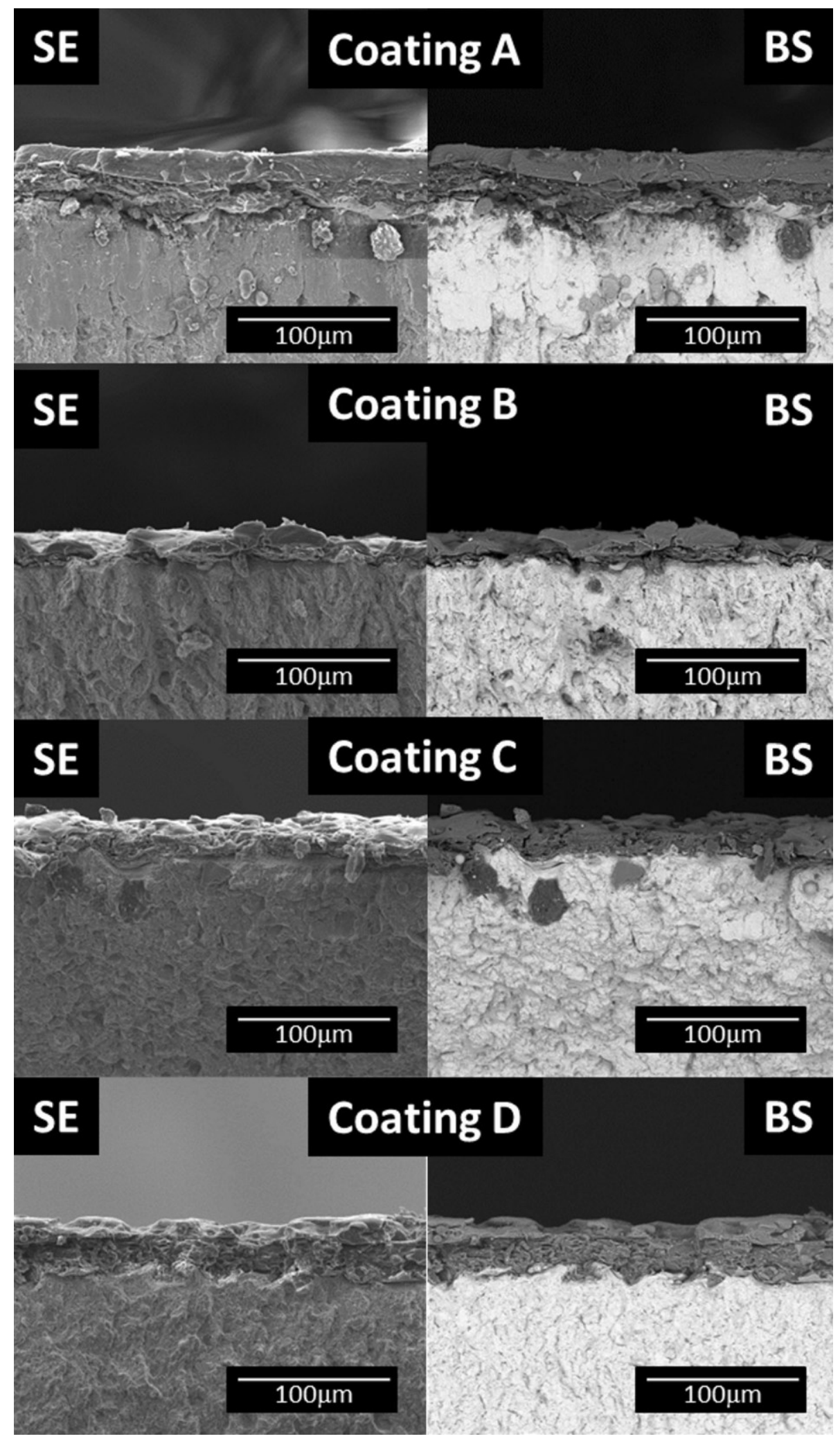

Fig. 8 Cross section of all coatings in unworn zone 


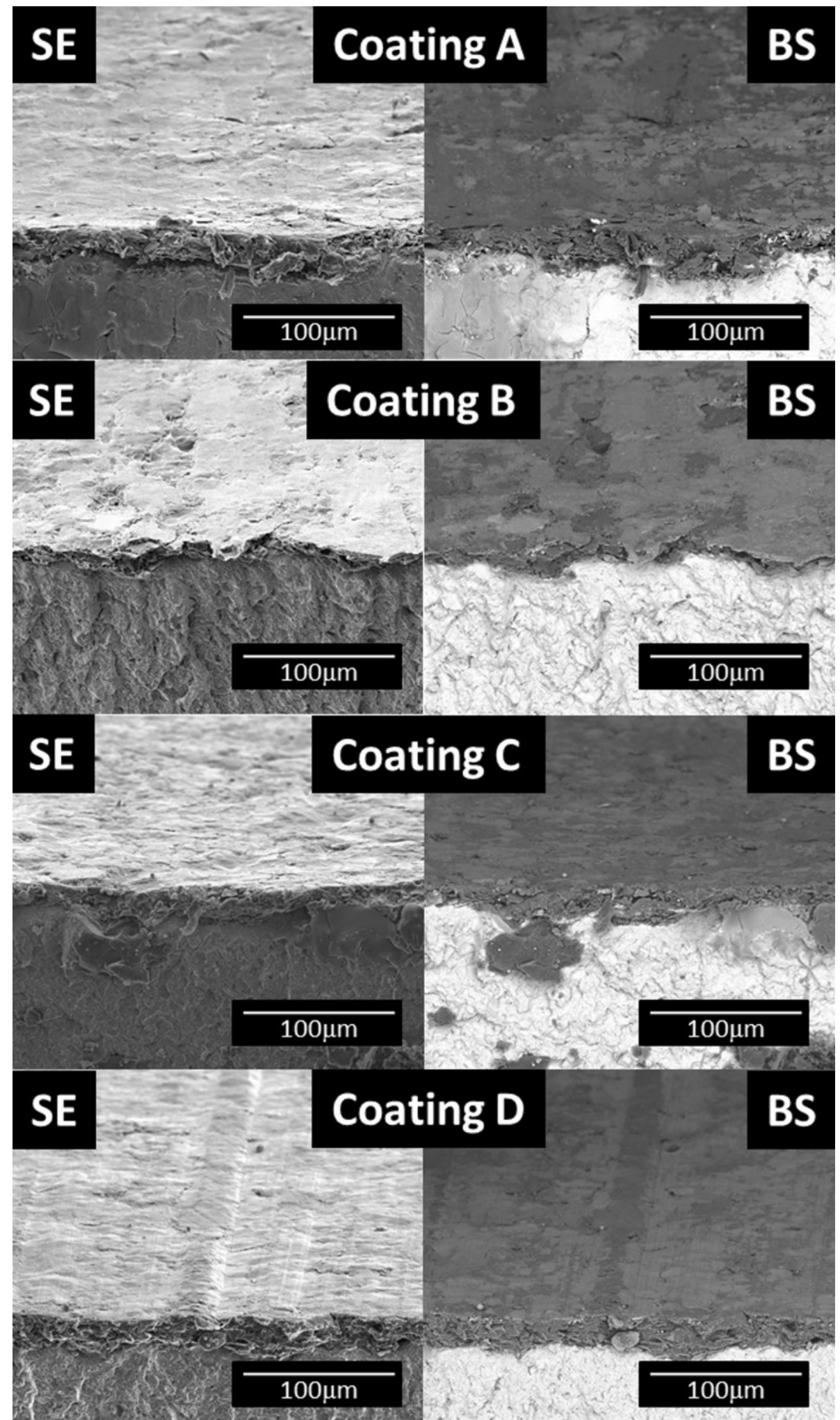

Fig. 9 Cross section of all coating in worn zone (after $2000 \mathrm{~m}$ sliding length) 

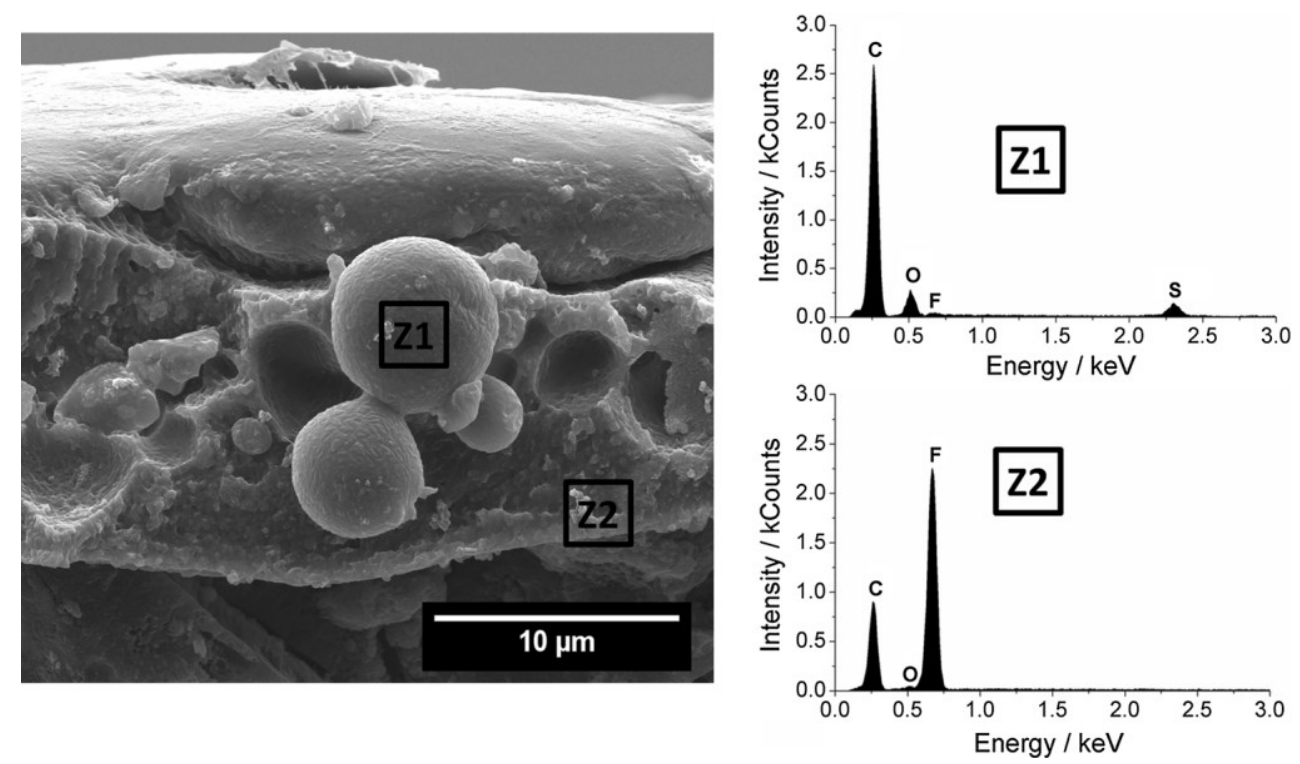

Fig. 10 Microcapsules incorporated into coating system and EDS spectra of microcapsules (Z1) and EDS spectra of coating (Z2) 

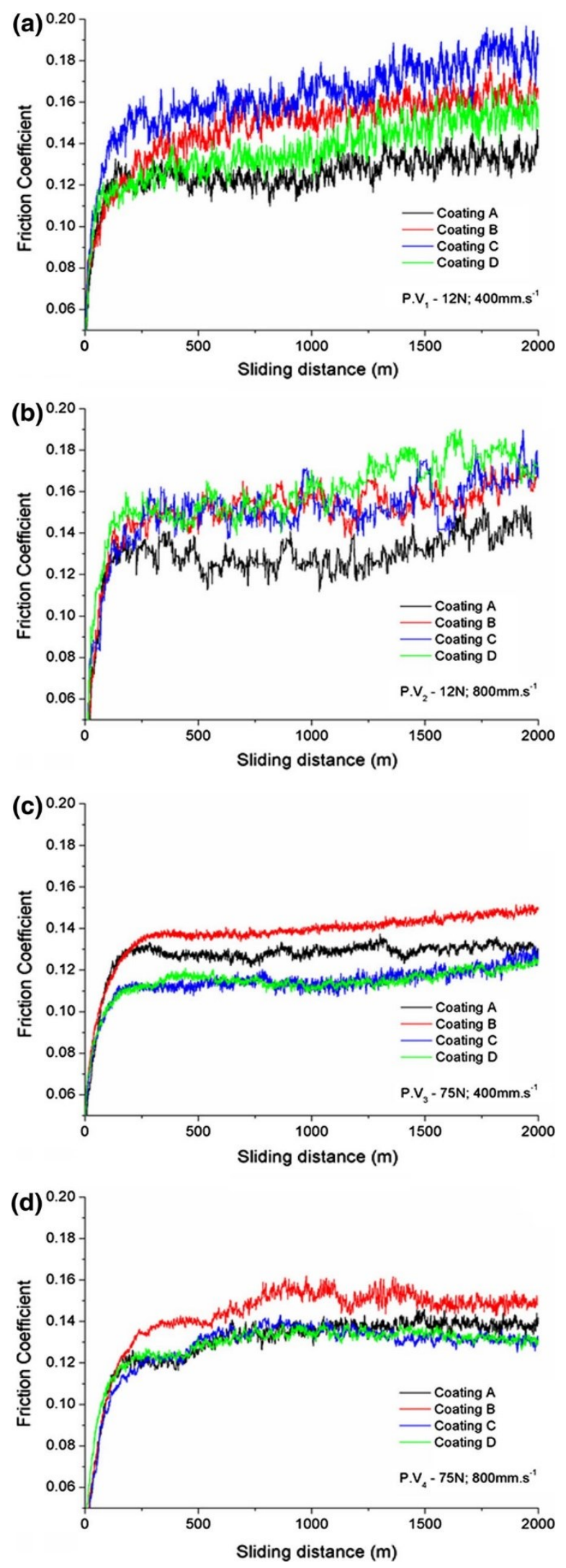

Fig. 11 Friction coefficient of coatings as a function of sliding length for all P.V tested. a $12 \mathrm{~N}$ at $400 \mathrm{~mm} \mathrm{~s}^{-1}$, b $12 \mathrm{~N}$ at $800 \mathrm{~mm} \mathrm{~s}^{-1}$, c $75 \mathrm{~N}$ at $400 \mathrm{~mm} \mathrm{~s}^{-1}, \mathrm{~d} 75 \mathrm{~N}$ at $800 \mathrm{~mm}$ $s^{-1}$ 


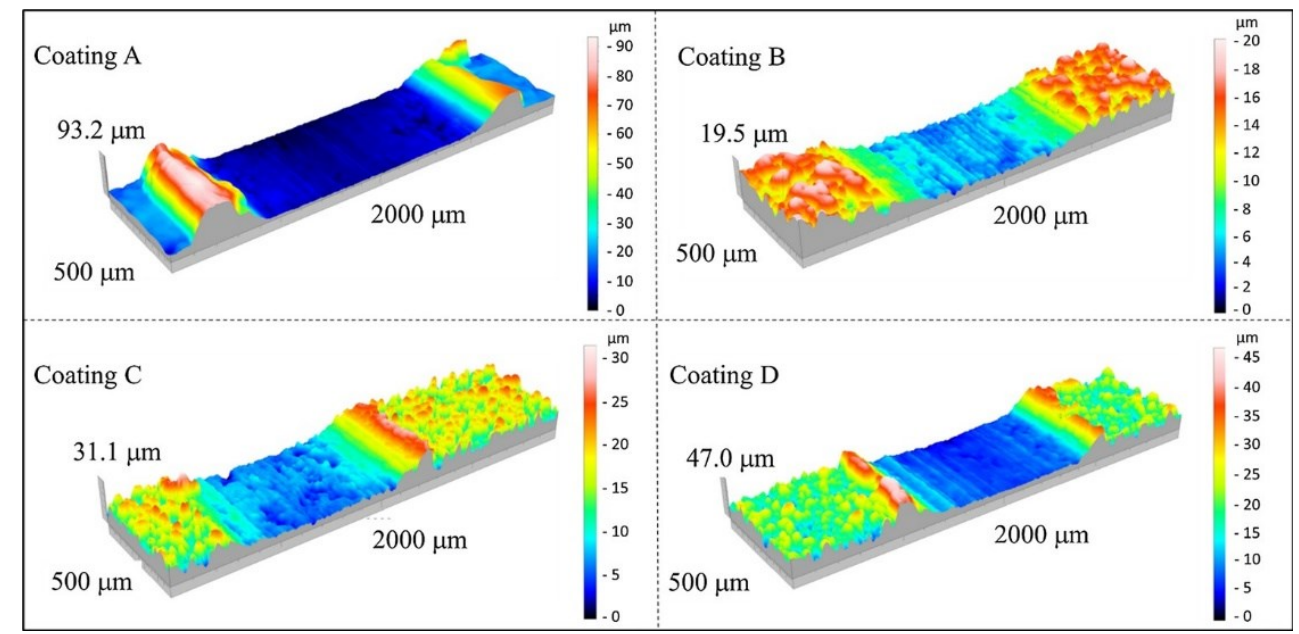

Fig. 12 Wear profile of coatings after tribological test for $75 \mathrm{~N}$ at $400 \mathrm{~mm} \mathrm{~s}^{-1}$

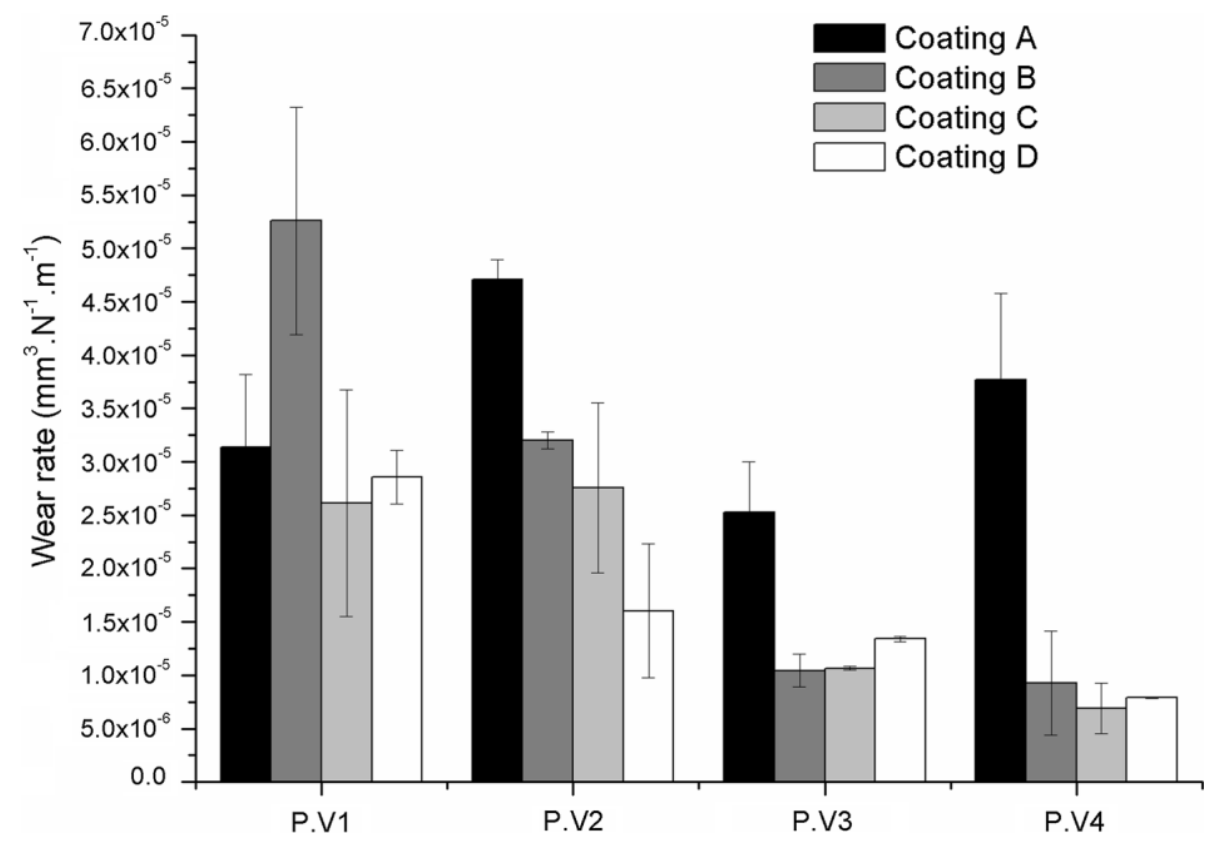

Fig. 13 Wear rate analysis for all coatings and all P.V conditions: P.V1 $=12 \mathrm{~N}$ at $400 \mathrm{~mm}$ $\mathrm{s}^{-1}, \mathrm{P} . \mathrm{V} 2=12 \mathrm{~N}$ at $800 \mathrm{~mm} \mathrm{~s}^{-1}, \mathrm{P} . \mathrm{V} 3=75 \mathrm{~N}^{2} 400 \mathrm{~mm} \mathrm{~s}^{-1}, \mathrm{P} . \mathrm{V} 3=75 \mathrm{~N}$ at $800 \mathrm{~mm}$ $\mathrm{s}-1$ 


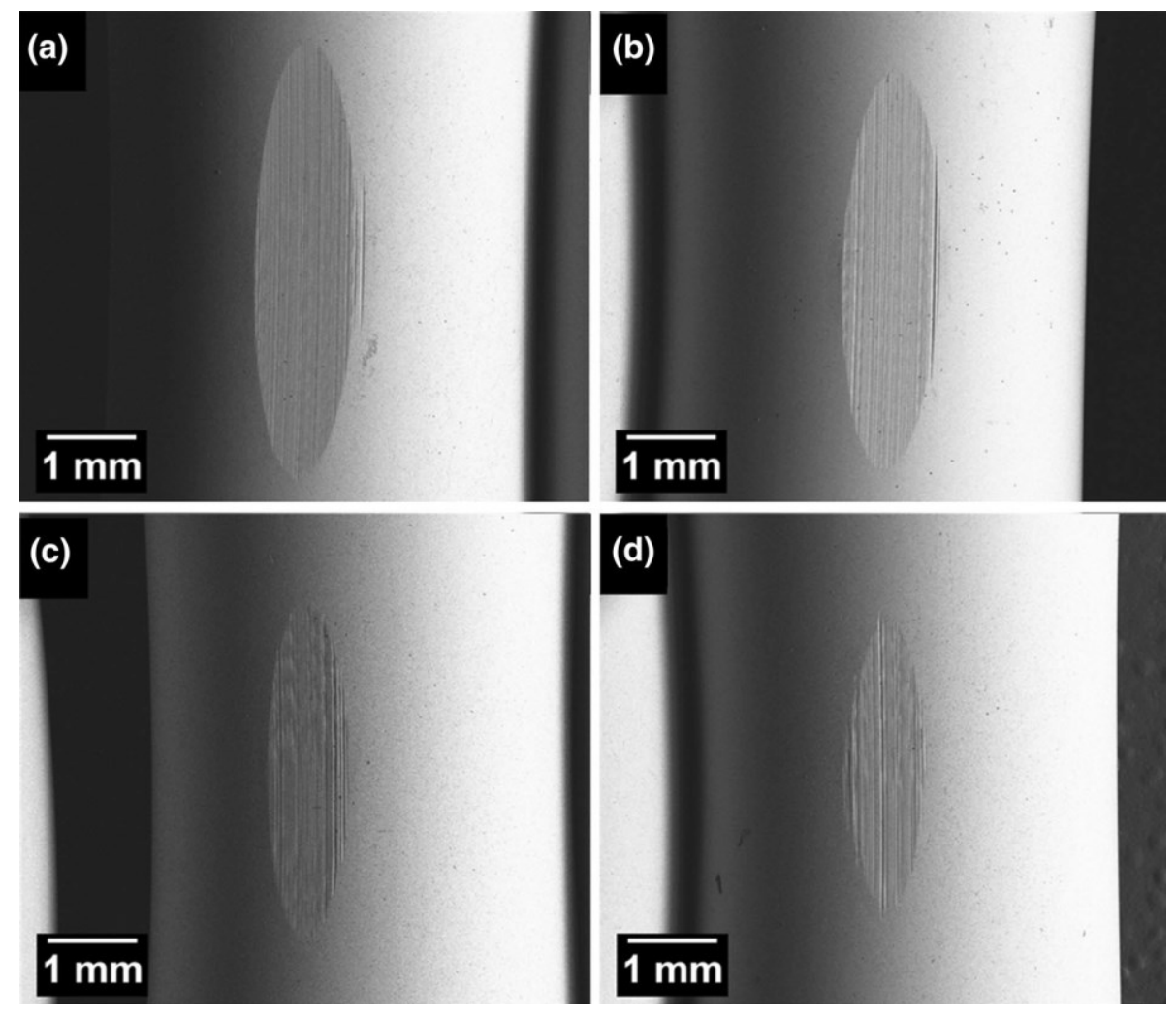

Fig. 14 SEM images of test counterfaces. Magnification: 409. a Coating A, b Coating B, c Coating C, d Coating D
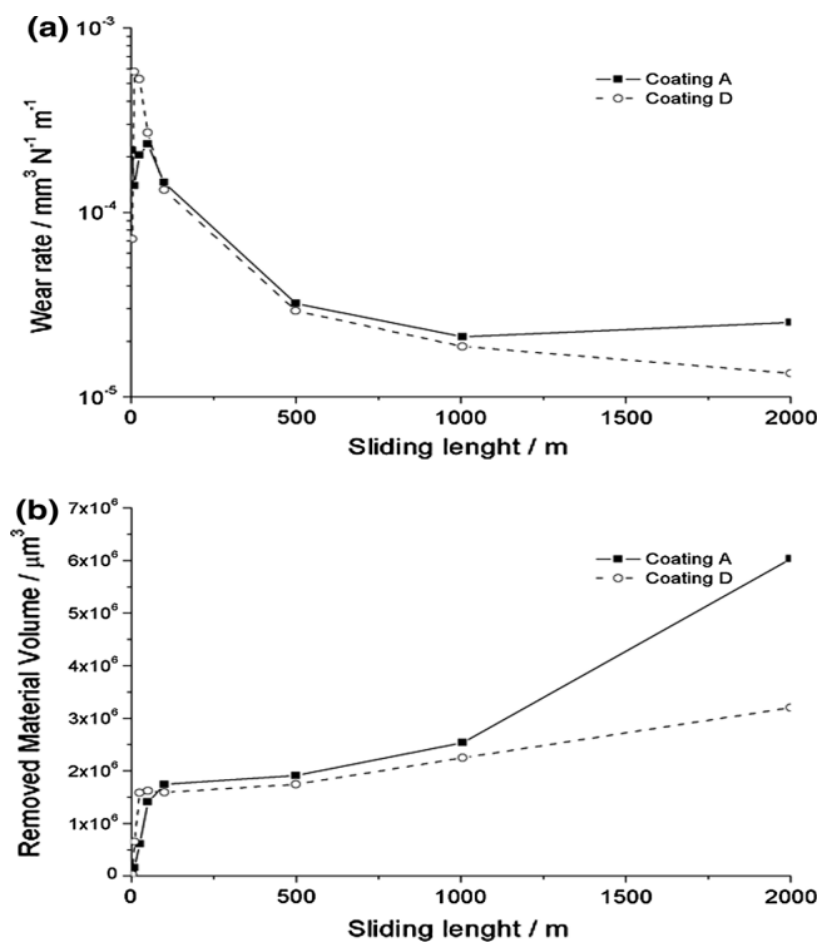

Fig. 15 Wear rate (a) and removed material volume (b) relative to sliding length for Coatings $\mathrm{A}$ and $\mathrm{D}$ and for $75 \mathrm{~N}$ at $400 \mathrm{~mm} \mathrm{~s}^{-1}$ test conditions 
Table 1 Load and speed conditions tested

\begin{tabular}{|c|c|c|}
\hline Designation & Load $(\mathrm{N})$ & Speed $\left(\mathrm{mm} \mathrm{s}^{-1}\right)$ \\
\hline P.V 1 & 12 & 400 \\
\hline P. $V_{2}$ & 12 & 800 \\
\hline P. $V_{3}$ & 75 & 400 \\
\hline P. $V_{4}$ & 75 & 800 \\
\hline
\end{tabular}

